\title{
Modified Ti/MCM-41 catalysts for enantioselective epoxidation of styrene
}

\author{
Marwa Fadhli, ${ }^{\mathrm{a}, *}$ Ilyes Khedher ${ }^{\mathrm{a}}$ and José M. Fraile ${ }^{\mathrm{b}, *}$ \\ ${ }^{a}$ Université de Tunis El-Manar, Faculté des Sciences de Tunis, Laboratoire de Chimie des \\ Matériaux et Catalyse, 2092Tunis, Tunisia. \\ ${ }^{b}$ Instituto de Síntesis Química y Catálisis Homogénea (ISQCH). Facultad de Ciencias, \\ C.S.I.C.-Universidad de Zaragoza, E-50009 Zaragoza, Spain.
}

\begin{abstract}
Ti sites grafted on MCM-41, pretreated at different temperatures, have been modified with chiral tartrates, leading to different species. Those solids are active catalyst for the epoxidation of styrene with tert-butyl hydroperoxide, although their efficiency is not optimal, mainly due to the subsequent epoxide rearrangement to phenyl acetaldehyde and further oxidation of it. Styrene oxide is obtained with moderate enantioselectivity, in the range of 55$62 \%$ e.e., and the best overall results correspond to the use of MCM-41 calcined at the lowest temperature $\left(550^{\circ} \mathrm{C}\right)$. These results are much better with regard to styrene oxide selectivity than those obtained in homogeneous phase under the same conditions, whereas enantioselectivity is similar. The heterogeneous catalysts are recoverable, but only partially due to a loss in activity, although enantioselectivity is kept at similar levels, demonstrating the stability of the supported Ti-tartrate species.
\end{abstract}

Keywords: Titanium; MCM-41; enantioselective epoxidation; styrene oxide

* Corresponding authors:

Marwa Fadhli, e-mail: marwafadhli@hotmail.fr

José M. Fraile, e-mail: jmfraile@unizar.es 


\section{Introduction}

Chiral epoxides have shown to be interesting compounds, useful as synthetic intermediates for the preparation of a large variety of organic compounds [1]. Asymmetric epoxidation of prochiral alkenes represents a powerful strategy for the synthesis of enantiomerically enriched epoxides. In this field, the most important way to generate optically active epoxides is via enantioselective catalysis. In the past three decades, there has been great progress in catalytic asymmetric alkene epoxidation. Probably, the most important methods for catalytic enantioselective epoxidation are the Ti-tartrate system for allylic alcohols with alkyl hydroperoxides [2] and the Mn-salen system for epoxidation of unfunctionalized alkenes [3], in both cases with excellent catalytic activity and enantioselectivity in a broad range of alkenes. Some of those catalysts have been immobilized on solids in an attempt to combine the activity and selectivity of the homogeneous catalysts and the practical advantages of heterogeneous catalysts, such as the better handling properties, and the simple separation and recycling of the catalyst, together with the easier purification of the products [4]. Various strategies have been reported to immobilize chiral transition metal complexes onto solid supports, including covalent grafting between the solid support and the chiral ligand [5], as well as alternative methods that do not involve the direct bonding of the chiral ligand [6]. Whereas a good number of successful examples have been described about the immobilization of chiral salen-metal complexes [7], this is not the case with Ti-tartrate catalysts [8]. Linear [9] and branched [10] polytartrates led to values of enantioselectivity (up to $80 \%$ ee) lower than the soluble tartrates in the epoxidation of allylic alcohols. A similar result in enantioselectivity (up to $86 \%$ ee) was obtained with tartramides supported on inorganic siliceous materials [11].

Titanium sites can be grafted on the silica surface through the formation of Si-O-Ti bonds by reaction with precursors such as $\mathrm{Ti}\left(\mathrm{O}^{\mathrm{i}} \mathrm{Pr}\right)_{4}[12]$ or $\mathrm{TiCp}_{2} \mathrm{Cl}_{2}$ [13], and this kind of solids has 
been successfully used in epoxidation reactions with either alkyl hydroperoxides [14] or hydrogen peroxide [15]. The residual Ti-OR (or Ti-OH) groups can be substituted by other alcohols, such as tartaric acid derivatives [16], which modifies the catalytic activity of the titanium sites $[17,18]$. In spite of the chiral nature of the silica-Ti-tartrate sites, only a limited number of examples have been described of application of this type of catalysts in enantioselective oxidations, either epoxidation [18,19] or sulfoxidation [20], with rather poor results.

The difficulty in reproducing the results in solution with allylic alcohols using heterogeneous catalysts may lay on the dimeric nature of the homogeneous catalyst, in which the titanium is in octahedral coordination, accommodating the bidentate ligand, the oxidant and the allylic alcohol [21]. Those catalysts with flexibility high enough to reach this kind of arrangement, such as polytartrates $[9,10]$, led to the best results. However, the grafted Ti sites with two alkoxides from tartrate ligand and at least one silyloxy grafting bond cannot accommodate at the same time the hydroperoxide and the allylic alkoxide in the epoxidation process. In contrast, analogous Ta-silica catalysts modified with tartrates have shown good performance in the epoxidation of allylic alcohols [22], as they can accommodate 5 alkoxide bonds, required for grafting, chiral ligand bonding and coordination of the two reagents. However, it would be conceivable the formation of a titanium species with one silyloxy bond to the surface, two alkoxide bonds with a chiral tartrate and one with the hydroperoxide, that would be able to epoxidize unfunctionalized alkenes. This kind of epoxidation reaction with homogeneous titanium catalysts has been only recently described using chiral salan ligands $[23,24]$ and hydrogen peroxide as the oxidant. In that case, the role of the N-H groups of the ligands has been proposed as crucial to stabilize the Ti-peroxo intermediate. 
In this manuscript, we describe the preparation of Ti-tartrate complexes grafted on the surface of MCM-41, and their use in the epoxidation of styrene with tert-butyl hydroperoxide, with special emphasis on the type of surface species formed.

\section{Experimental}

\subsection{Synthesis}

MCM-41 was prepared as described elsewhere [25]. In order to remove the organic surfactant (cetyltrimethylammonium bromide) from the pores of MCM-41, the dried as-synthesized samples were calcined in air at three different temperatures 550,650 and $750^{\circ} \mathrm{C}$ for $6 \mathrm{~h}$, using a heating rate of $1{ }^{\circ} \mathrm{C} / \mathrm{min}$. The resulting materials are denoted as $\mathrm{MCM}_{550}, \mathrm{MCM}_{650}$ and $\mathrm{MCM}_{750 .}$

Titanium (IV) tetraisopropoxide $\left(\mathrm{Ti}\left(\mathrm{O}^{i} \mathrm{Pr}\right)_{4}\right)$ was used as a titanium precursor. A mixture of $\operatorname{Ti}\left(\mathrm{O}^{i} \mathrm{Pr}\right)_{4}(0.4 \mathrm{mmol})$ and calcined MCM support $(1 \mathrm{~g})$ in dry toluene $(10 \mathrm{~mL})$ was refluxed for $24 \mathrm{~h}$ under inert atmosphere and the resulting material was vacuum-filtered, washed with anhydrous dichloromethane and dried overnight in an oven at $120^{\circ} \mathrm{C}$. Dried materials were subjected to Soxhlet extraction with anhydrous dichloromethane for $24 \mathrm{~h}$ and the final solids were dried at $50^{\circ} \mathrm{C}$ under vacuum for $8 \mathrm{~h}$. The resulting catalysts are referred to as Ti$\mathrm{MCM}_{550}, \mathrm{Ti}-\mathrm{MCM}_{650}$ and Ti-MCM 750.

Diethyl L-tartrate or diisopropyl L-tartrate $(0.4 \mathrm{mmol})$ was added to a suspension of Ti-MCM $(1 \mathrm{~g})$ in dry toluene $(20 \mathrm{ml})$, and the mixture was refluxed for $12 \mathrm{~h}$ under inert atmosphere. The resulting solids were filtered and thoroughly washed with dry dichloromethane and diethyl ether to eliminate the physisorbed chiral ligand. The solids were dried at $120^{\circ} \mathrm{C}$ overnight and kept in a desiccator until use. These catalysts are referred to as DET-Ti-MCM or DIPT-Ti-MCM. 


\subsection{Characterization methods}

Powder small angle X-ray diffraction patterns were recorded using $\mathrm{Cu} \mathrm{K} \alpha$ radiation $(40 \mathrm{kV}$ and $30 \mathrm{~mA}$ ) from $2 \theta=1^{\circ}$ up to $2 \theta=10^{\circ}$. BET surface area and pore size distribution were determined from the $\mathrm{N}_{2}$ adsorption-desorption isotherms at $77 \mathrm{~K}$ using a micrometrics ASAP 2000 apparatus. Infrared spectra of samples were recorded in the range of $4000-400 \mathrm{~cm}^{-1}$ with discs of solids mixed with dry $\mathrm{KBr}$ on a Perkin-Elmer FT-IR paragon 1000PC spectrometer. The titanium content was carried out by plasma atomic emission spectroscopy (ICP-OES) using a Thermo Elemental IRIS Intrepid spectrometer. Diffuse reflectance UV-Vis (DR-UV) spectra were recorded on a Unicam UV4 spectrometer equipped with a labsphere diffuse reflectance attachment. Elemental analysis (CHNS) was performed on a Perkin Elmer 2400 elemental analyzer. MAS NMR spectra were recorded in a Bruker Avance III WB400 spectrometer with $4 \mathrm{~mm}$ zirconia rotors spun at magic angle in $\mathrm{N}_{2}$ at $10 \mathrm{kHz} .{ }^{1} \mathrm{H}-{ }^{13} \mathrm{C} \mathrm{CP}$ spectra (12000-20000 scans) were measured using a ${ }^{1} \mathrm{H} \pi / 2$ pulse length of $3 \mu$ s, with a contact time of $3 \mathrm{~ms}$, and spinal64 proton decoupling sequence of $5.3 \mu$ s pulse length, with $7 \mathrm{~s}$ recycle delay.

\subsection{Catalytic test: styrene epoxidation}

To a solution of styrene $(10 \mathrm{mmol})$ in acetonitrile $(10 \mathrm{~mL})$, the catalyst $(0.015 \mathrm{mmol}$ of $\mathrm{Ti}, 40$ $\mathrm{mg}$ in the case of solid catalysts) and TBHP (6 mmol, 5.5 solution in decane) were added. The mixture was allowed to stir at $70^{\circ} \mathrm{C}$ under inert atmosphere. The reaction was monitored by $\mathrm{GC}$ and at the end the solid was separated by filtration, thoroughly washed with $\mathrm{CH}_{2} \mathrm{Cl}_{2}$ and then dried at $120^{\circ} \mathrm{C}$ under vacuum to be reused. The enantiomeric excess was determined by HPLC with a Chiralpack OD-H column. 


\section{Results and discussion}

\subsection{Preparation and characterization of the catalysts}

As commented above, the possibility of using grafted Ti-tartrate species requires a certain control on the type of species formed on the silica surface. Depending on the surface density of $\mathrm{Si}-\mathrm{OH}$ groups, the distribution of the resulting metal surface species (monopodal, bipodal or tripodal, bonded to one, two or three silicon atoms through $\mathrm{Si}-\mathrm{O}-\mathrm{Ti}$ bonds, respectively, Figure 1) will be different. This will condition the type of chiral species formed after treatment with a chiral tartrate (Figure 1), with the tripodal species being the less interesting one, whereas the monopodal species would lead easily to the active intermediate by substitution of the remaining isopropoxide. Thus, the siliceous MCM-41 was pre-calcined at different temperatures, 550,650 and $750^{\circ} \mathrm{C}$, before titanium grafting.
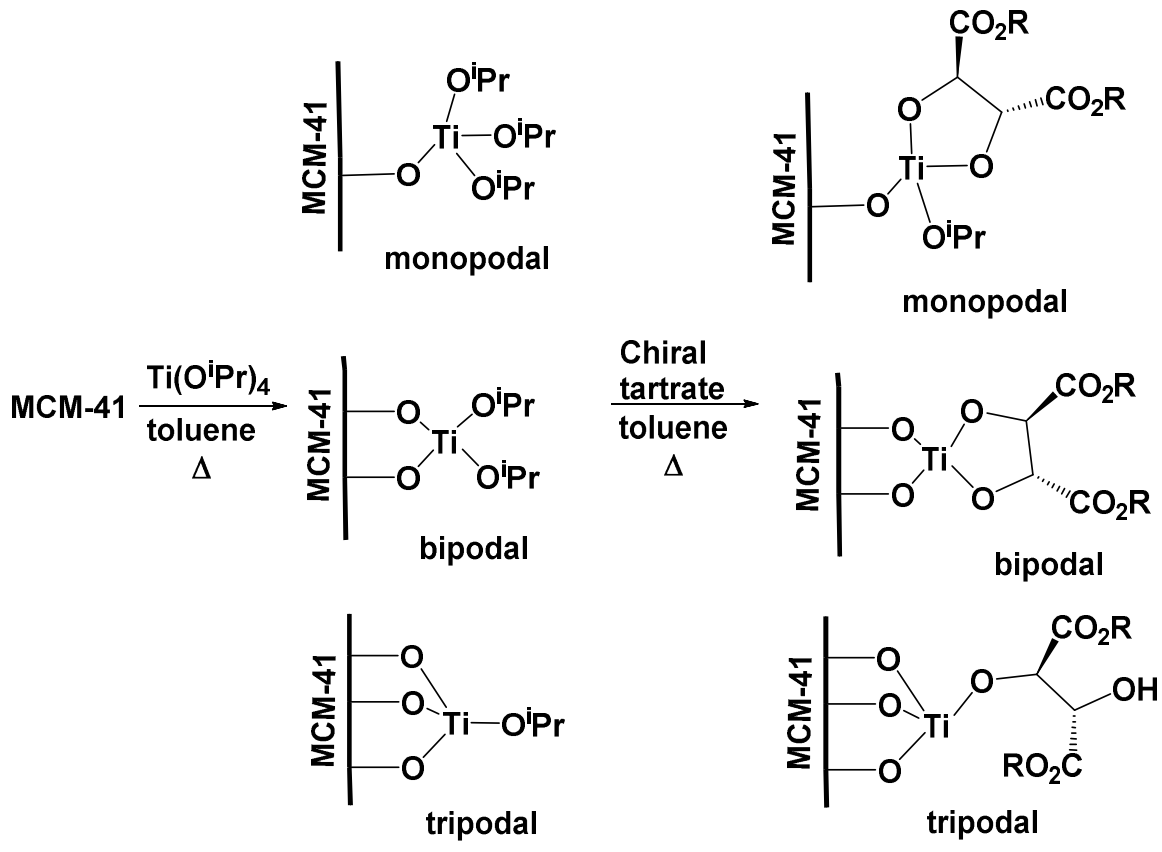

Figure 1. Possible surface Ti species.

The low-angle XRD patterns recorded on untreated $\mathrm{MCM}_{550}, \mathrm{MCM}_{650}$ and $\mathrm{MCM}_{750}$ samples match well with those of the silica MCM-41 polymorph reported by Chen et al [26]. The three diffraction peaks in the $2 \theta$ range of $1-10^{\circ}$, characteristic of the MCM-41 structure and corresponding to the (100), (110) and (200) reflections [27], were reasonably well resolved 
(Fig. S4). The diffraction peaks did not significantly change after calcination at $750^{\circ} \mathrm{C}$ except a slightly lower intensity, which indicates that the hexagonal structure of MCM41 is preserved even after calcination at $750^{\circ} \mathrm{C}$. The treatment with $\mathrm{Ti}\left(\mathrm{O}^{i} \mathrm{Pr}\right)$ produces a decrease in the intensities of all of the peaks with a little shift toward lower $2 \theta$ values. Figure 2 shows the XRD patterns of $\mathrm{MCM}_{550}$ samples, as an example. This indicates that the incorporation of Ti species in the channel of MCM-41 leads to a substantial loss in the scattering contrast between the channel and the wall, and then reduces the intensity of the scattered X-ray in the powder diffraction experiment [28].

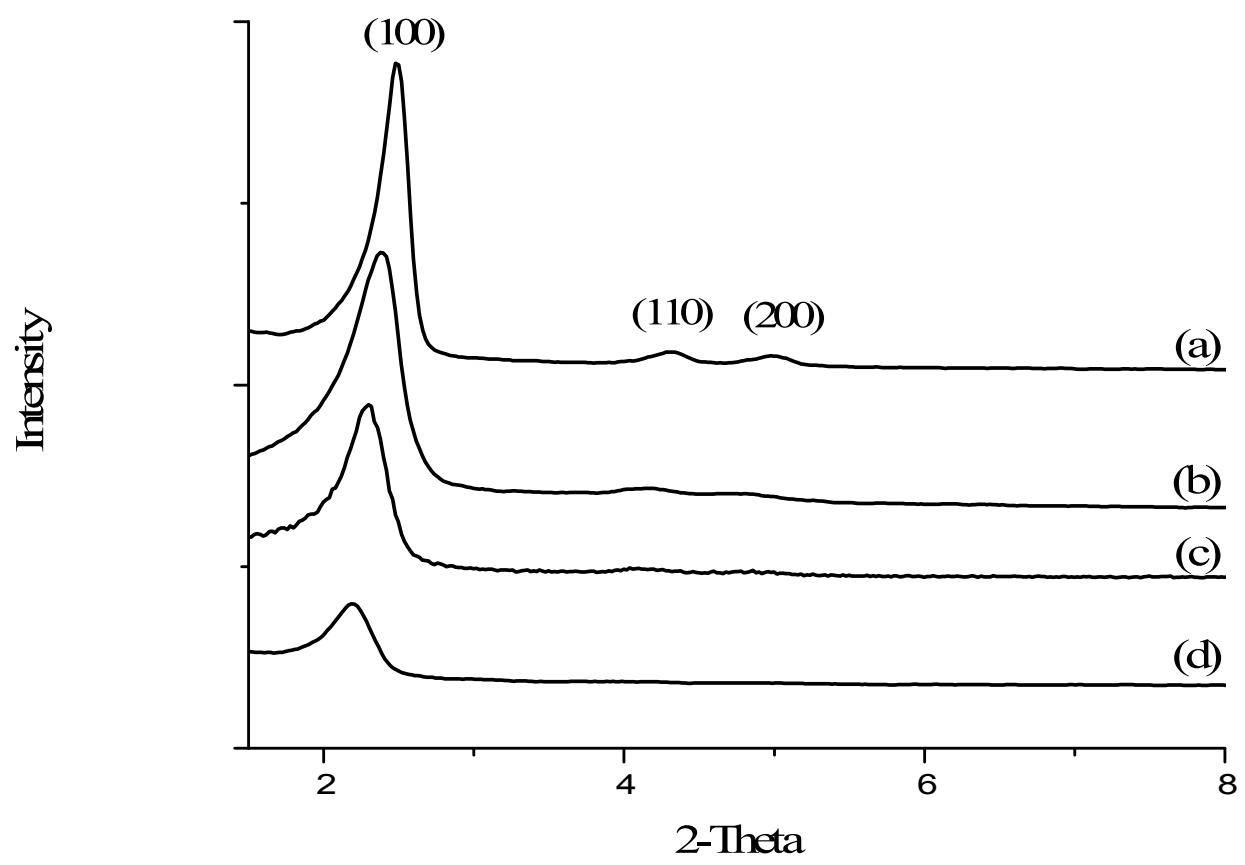

Figure 2. Low-angle XRD patterns of different mesoporous solids: a) $\mathrm{MCM}_{550}$, b) Ti-

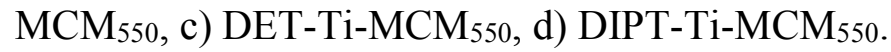

When R-(+)-diethyl L-tartrate was added (Fig. 2c), the intensities of all the peaks decrease even more, notably the intensity of the two less intense reflections (110) and (200), indicating a loss of the long-range regularity of the hexagonal arrays of mesopores of MCM-41. This effect is more obvious in the case of DIPT-Ti-MCM, showing the disappearance of (110) and (200) peaks, due to the larger volume of R-(+)-diisopropyl L-tartrate (Fig. 2d). As shown in Table 1, $a_{0}$ unit-cell parameter of DIPT-Ti-MCM and DET-Ti-MCM samples increase much 
more compared to Ti-MCM samples. In the high angle region of XRD patterns, the main diffraction peak corresponding to the bulk $\mathrm{TiO}_{2}\left(2 \theta=25.5^{\circ}\right)$ did not appear for both treated MCM-41samples. This suggests that Ti is well dispersed within silica channels.

The nitrogen adsorption-desorption isotherm of the untreated MCM-41 sample follows the type-IV isotherm and shows a sharp capillary condensation step at a relative pressure between 0.2 and 0.4 , indicating a typical mesoporous structure with uniform pores (see Fig. 3) [29]. After treatment, the sharp capillary condensation step shifts toward lower $\mathrm{P} / \mathrm{P}_{0}$ region. This suggests that the introduction of $\mathrm{Ti}(\mathrm{OiPr})_{4}$ with DET or DIPT into the channel causes changes in the pore structure of the support during the incorporation process. These changes could be observed more clearly in Table 1 . When MCM-41was treated with $\operatorname{Ti}\left(\mathrm{O}^{\mathrm{i}} \mathrm{Pr}\right) 4$, and even more after modification with chiral tartarates, the BET surface area, pore volume and pore diameter decrease, while the wall thickness $\left(\delta\right.$, estimated as $\left.\delta=\mathrm{a}_{0}-\mathrm{D}\right)$ increases. This result is in good agreement with the grafting of Ti species on the silica inner surface, increasing in this way the wall thickness. Consequently, the mesochannels became narrow and the pore diameter of the incorporated MCM41 samples would also decrease accompanying the reduction of the pore volume and the surface area.

The average amount of isopropoxy ligands remaining bound to titanium sites on the surface can be estimated by the quantification of the carbon-to-titanium ratio obtained by elemental analysis (Table 1). In the case of Ti-MCM41550, isopropoxy/titanium molar ratio is around 1.7 whereas in Ti-MCM41 650 and Ti-MCM41 750 the value is higher, 2.4 and 2.3, respectively. In all the solids, the major surface titanium species seems to be the bipodal one (isopropoxy/titanium $=2$ ), with a larger contribution of tripodal species (Figure 1) in TiMCM41550, in agreement with its higher silanol density due to the lower calcination temperature. The effect is the opposite in the other two solids calcined at higher temperature, which should present a lower silanol density and hence a larger contribution of the monopodal 
species (isopropoxy/titanium $=3$ ). When tartrate was added (DET or DIPT), an increase of $\mathrm{C} / \mathrm{Ti}$ in all cases was detected indicating the adsorption of tartrate. Carbon analyses are compatible with a tartrate/Ti ratio in the order of 1.2-1.6 in the case of DET and 1.1-1.4 in the case of DIPT. However, these values must be taken with care, as monopodal species (Figure 1) must keep one isopropoxy group $(\mathrm{C} / \mathrm{Ti}=11$ with $\mathrm{DET}$ and 13 with $\mathrm{DIPT})$. Other sources of inaccuracy of the analysis would be the inclusion of a second molecule of tartrate as monodentate ligand to substitute the remaining isopropoxy group in monopodal species, or the physisorption of tartrate on the silica surface of MCM41.

The FT-IR spectra of the calcined MCM41 samples (Figure 5a shows the spectrum of $\mathrm{MCM}_{550}$, as an example) show the typical bands for a silica based solid. The most important bands are the O-H stretching of the hydrogen-bound silanol groups (large broad band, 3700$\left.3000 \mathrm{~cm}^{-1}\right)$, the deformation vibrations of absorbed water molecules $\left(1630 \mathrm{~cm}^{-1}\right)$, the asymmetric stretching vibration mode of the Si-O-Si linkage $\left(1300-1100 \mathrm{~cm}^{-1}\right)$, the Si-O stretching of the silanol groups $\left(960 \mathrm{~cm}^{-1}\right)$, and the symmetric stretching of $\mathrm{Si}-\mathrm{O}-\mathrm{Si}(800$ $\left.\mathrm{cm}^{-1}\right)$ [30]. After treatment with $\mathrm{Ti}\left(\mathrm{O}^{i} \mathrm{Pr}\right)_{4}$, some weak bands at $3000-2800 \mathrm{~cm}^{-1}$ are present, corresponding to the aliphatic $\mathrm{C}-\mathrm{H}$ bonds of the remaining isopropoxy groups. In the case of DET-Ti-MCM550 and DIPT-Ti-MCM550 samples (Figure $5 \mathrm{c}$ and d), a new peak appears at $1731 \mathrm{~cm}^{-1}$, corresponding to the $\mathrm{C}=\mathrm{O}$ bond of carboxylic esters. Its position [21] is in good agreement with the uncoordinated esters in the Ti(DIPT) complexes (spectra in solution, 1738 $\mathrm{cm}^{-1}$ ). In case that some of the esters in the solid were coordinated to Ti, their position in the spectrum $\left(1638 \mathrm{~cm}^{-1}\right)$ would be masked by the band corresponding to water molecules. 


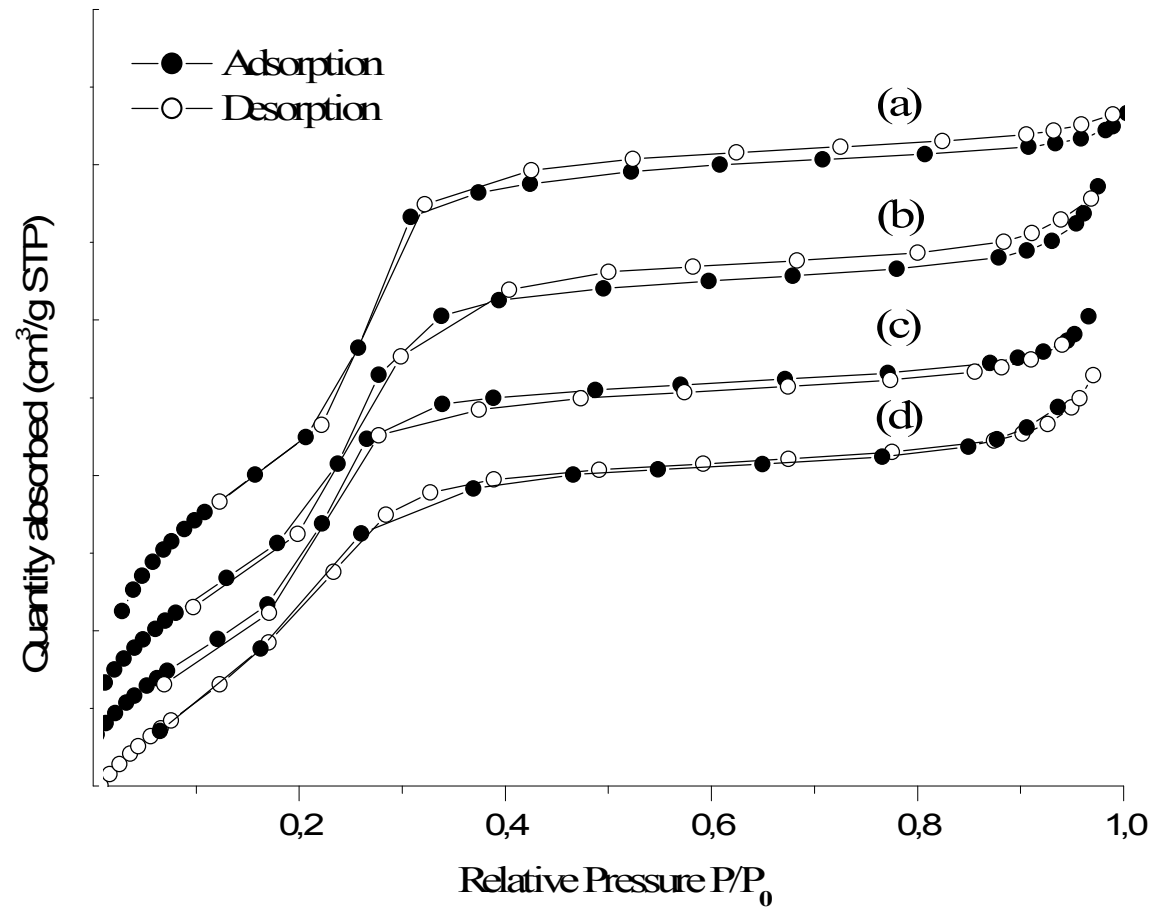

Figure 3. $\mathrm{N}_{2}$ adsorption-desorption isotherms at $77 \mathrm{~K}$ of the different mesoporous solids: a) $\left.\mathrm{MCM}_{550}, \mathrm{~b}\right)$ Ti-MCM550, c) Ti-MCM550-DET, d) Ti-MCM550-DIPT.

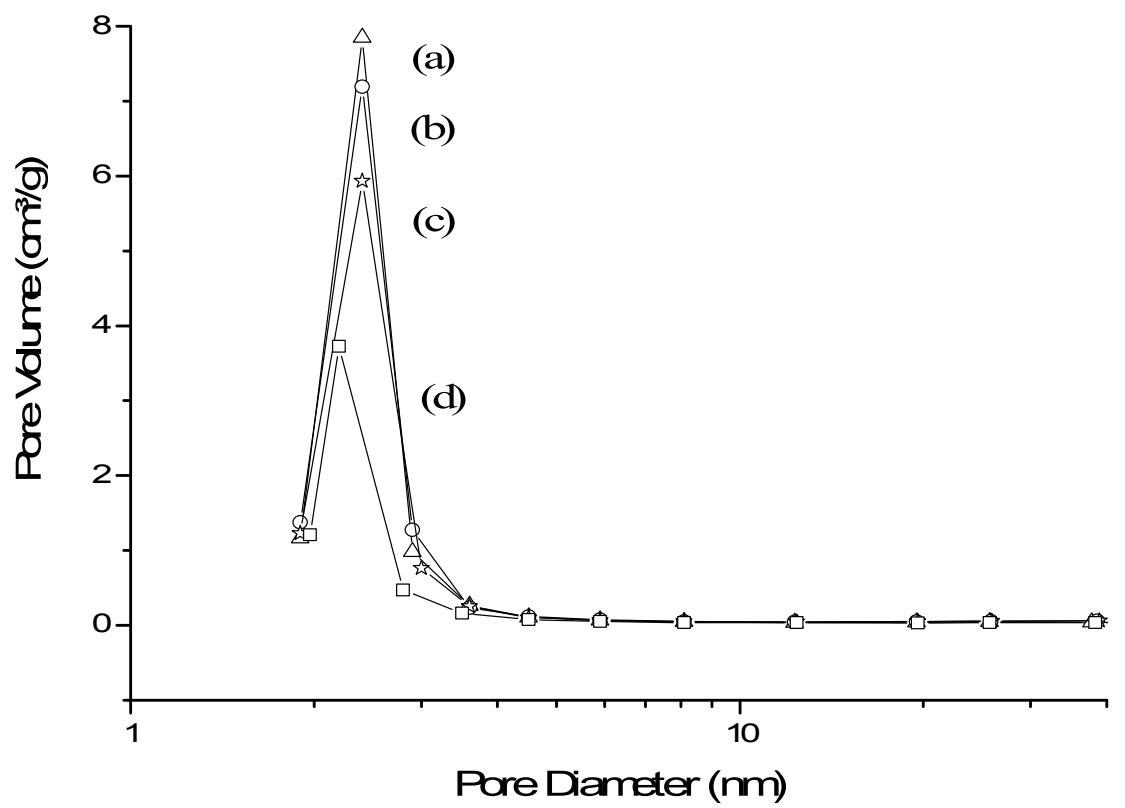

Figure 4. BJH pore size distribution curve of the different mesoporous solids: a) $\mathrm{MCM}_{550}$, b) Ti-MCM 550, c) Ti-MCM $550-D E T$, d) Ti-MCM 550 -DIPT. 
Table 1. Textural properties and elemental analysis of the titanium catalysts. ${ }^{\mathrm{a}}$

\begin{tabular}{|c|c|c|c|c|c|c|c|c|c|}
\hline Sample & $\mathrm{S}_{\text {BET }}\left(\mathrm{m}^{2} / \mathrm{g}\right)$ & $V_{p}\left(\mathrm{~cm}^{3} / \mathrm{g}\right)$ & $\mathrm{D}_{\mathrm{p}}(\mathrm{nm})$ & $\mathrm{d}_{100}(\mathrm{~nm})$ & $\mathrm{a}_{0}(\mathrm{~nm})$ & $\delta(\mathrm{nm})$ & $\mathrm{Ti}(\mathrm{mmol} / \mathrm{g})$ & $\mathrm{C}(\mathrm{mmol} / \mathrm{g})$ & $\mathrm{C} / \mathrm{Ti}$ \\
\hline $\mathrm{MCM}_{550}$ & 1001 & 0.76 & 3.03 & 3.55 & 4.10 & 1.07 & - & - & - \\
\hline $\mathrm{MCM}_{650}$ & 945 & 0.75 & 2.87 & 3.52 & 4.07 & 1.2 & - & - & - \\
\hline $\mathrm{MCM}_{750}$ & 925 & 0.64 & 2.74 & 3.24 & 3.74 & 1.0 & - & - & - \\
\hline Ti-MCM550 & 966 & 0.75 & 2.99 & 3.73 & 4.31 & 1.32 & 0.38 & 1.97 & 5.18 \\
\hline $\mathrm{Ti}^{-\mathrm{MCM}_{650}}$ & 921 & 0.61 & 2.66 & 3.67 & 4.24 & 1.58 & 0.38 & 2.79 & 7.33 \\
\hline Ti-MCM750 & 814 & 0.57 & 2.35 & 3.47 & 4.01 & 1.66 & 0.38 & 2.61 & 6.86 \\
\hline DET-Ti- MCM550 & 804 & 0.65 & 2.56 & 3.87 & 4.46 & 1.9 & 0.38 & 3.62 & 9.52 \\
\hline DET-Ti- MCM 650 & 820 & 0.55 & 2.62 & 3.78 & 4.37 & 1.75 & 0.38 & 4.24 & 11.15 \\
\hline DET-Ti- MCM 750 & 730 & 0.52 & 2.34 & 3.64 & 4.21 & 1.87 & 0.38 & 4.9 & 12.89 \\
\hline DIPT-Ti- MCM 550 & 928 & 0.65 & 2.80 & 4.01 & 4.63 & 1.83 & 0.38 & 4.23 & 11.13 \\
\hline DIPT-Ti- MCM 650 & 802 & 0.53 & 2.64 & 3.83 & 4.43 & 1.79 & 0.38 & 5.18 & 13.63 \\
\hline DIPT-Ti- MCM 750 & 788 & 0.49 & 2.04 & 3.73 & 4.31 & 2.27 & 0.38 & 4.31 & 11.34 \\
\hline
\end{tabular}

${ }^{a} \mathrm{~S}_{\mathrm{BET}}=$ surface area; $\mathrm{V}_{\mathrm{p}}=$ pore volume; $\mathrm{D}_{\mathrm{p}}=$ mean pore diameter (determined by the BJH (Barrett-Joyner-Halenda) method); $\mathrm{d}_{100}=\lambda /(2 \sin \theta) ; \mathrm{a}_{0}=2 \mathrm{~d} \mathrm{~d}_{100} /$ $\sqrt{ } 3 ; \delta=$ mean wall thickness estimated according to $\delta=\mathrm{a}_{0}-\mathrm{D}_{\mathrm{p}}$. Ti content determined by ICP-OES. C content determined by elemental analysis. 

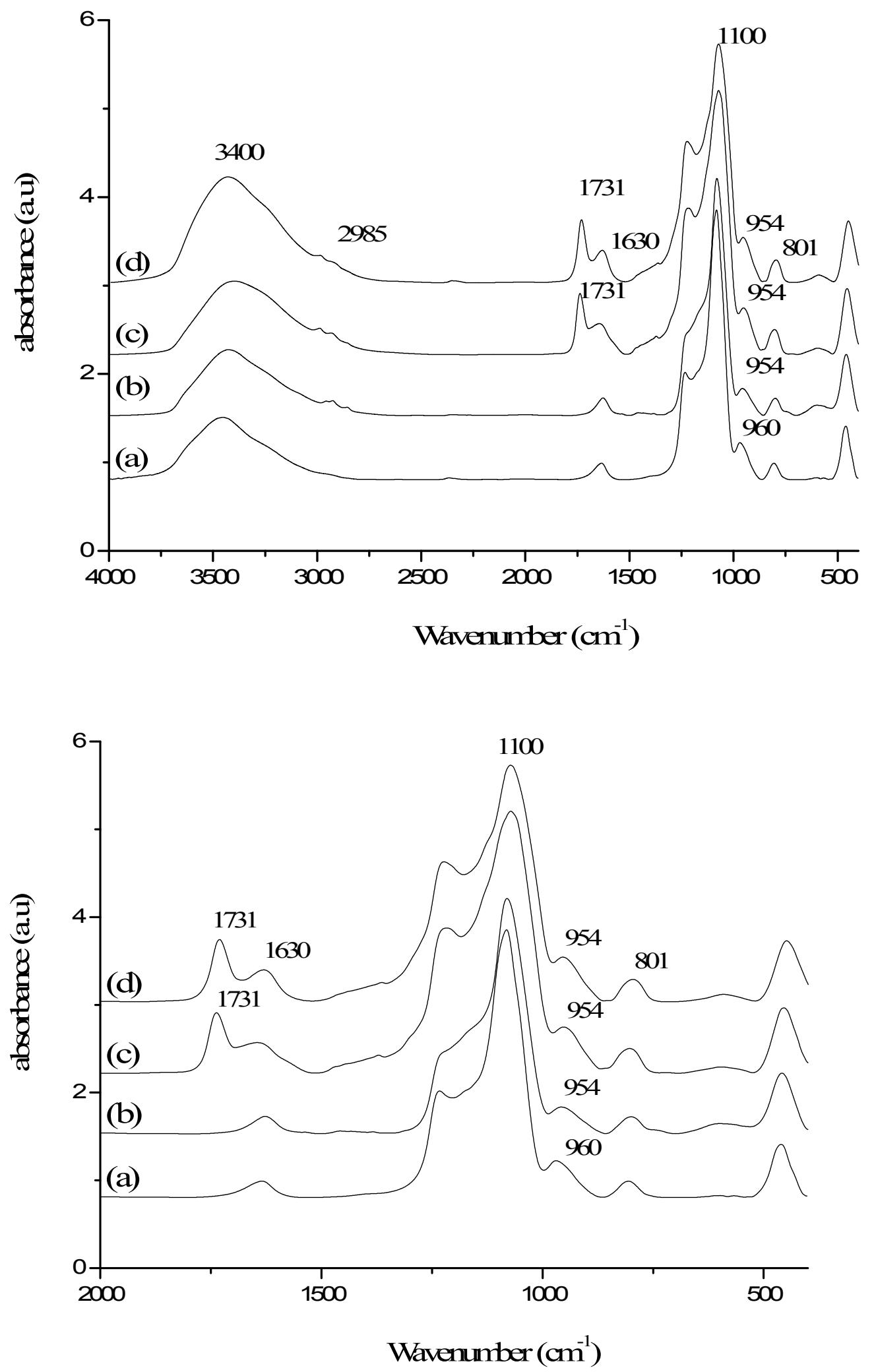

Figure 5. FT-IR spectra of a) $\mathrm{MCM}_{550}$, b) Ti-MCM ${ }_{550}$, c) DET-Ti-MCM ${ }_{550}$, d) DIPT-Ti$\mathrm{MCM}_{550 .}$ 
The formation of Ti-tartrate complexes on the solid surface was also checked by ${ }^{13} \mathrm{C}$ solid state NMR (Figure 6). The spectrum of DET physisorbed on purely siliceous MCM750 was recorded for the sake of comparison. The expected four narrow bands were observable, but with low intensity in the case of the carbonyl and the methyl signals due to the poor crosspolarization behavior, because of the lack of protons in the former, and because the easy rotation in the case of the methyl group. In all the Ti containing solids, the tartrate bands are wider, in agreement with a reduced mobility of the molecule, and probably by a broader distribution of different sites on the solid. The assignment of the signals can be done on the basis of the spectra of Ti-tartrate complexes in solution [21,31], as well as the solid state NMR spectra of complexes with different nuclearity $\left(\mathrm{Ti}_{3}(\mathrm{DIPT})_{3}\right.$ and $\left.\mathrm{Ti}_{4}(\mathrm{DIPT})_{4}\right)$ [32]. The two signals at 183 and $171 \mathrm{ppm}$ fit well with the presence of both coordinated and uncoordinated carboxylate groups. This possibility is confirmed by the presence of at least two different OEt groups in the case of DET-Ti-MCM, and even more types of OiPr groups are observable in DIPT-Ti-MCM, but the remaining groups from the starting $\operatorname{Ti}\left(\mathrm{O}^{\mathrm{i}} \mathrm{Pr}\right)_{4}$ may contribute to these signals. In the same way, the signal at 84 ppm clearly indicates the formation of $\mathrm{CH}-\mathrm{O}-\mathrm{Ti}$ bonds, whereas the presence of the signal at $72 \mathrm{ppm}$ points to the presence of free $\mathrm{CHOH}$ groups. This is probably due to a coordination of tartrate in a nonchelating way, as represented for tripodal species in Figure 1, and as pointed in the case of Ta catalysts [33]. 


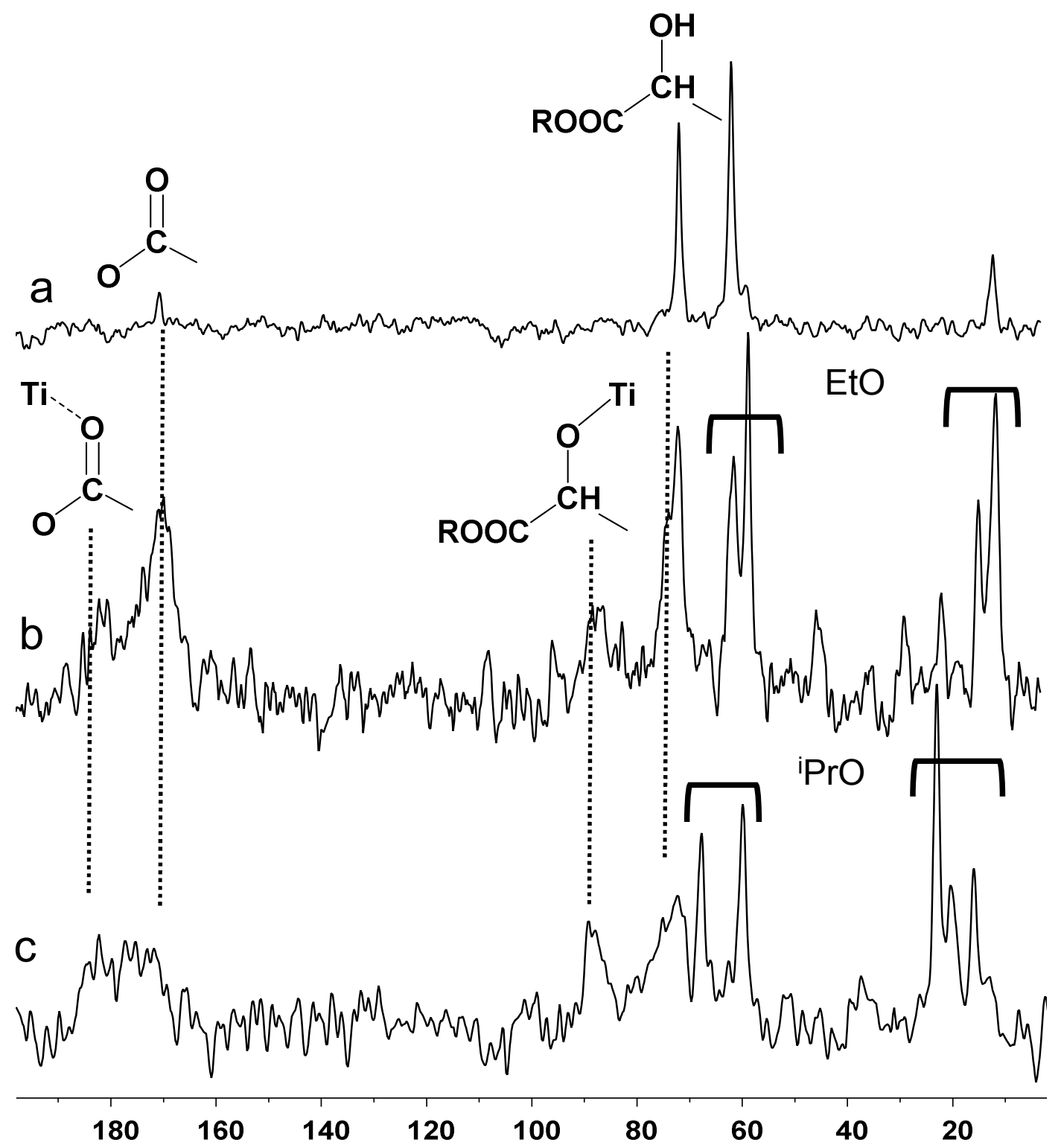

Figure $6 .{ }^{13} \mathrm{C}$ CP-MAS-NMR spectra of: a) DET physisorbed on $\mathrm{MCM}_{750}$, b) DET-Ti$\left.\mathrm{MCM}_{750}, \mathrm{c}\right)$ DIPT-Ti-MCM750

\subsection{Titanium catalyzed epoxidation of styrene}

One of the problems in the titanium catalyzed epoxidation of styrene is the existence of consecutive reactions (Scheme 1), namely the Meinwald rearrangement of the styrene oxide mainly to phenyl acetaldehyde (acetophenone is the minor product of this reaction), and the further oxidation of the former to phenyl acetic acid, which suffers an oxidative 
decarboxylation to benzaldehyde [34]. In view of that, and in an attempt to minimize the overoxidation of the products, tert-butyl hydroperoxide (TBHP) was used always in defect (0.6 equivalents) with respect to styrene, and the conversion and styrene oxide yield (Table 2) were calculated with respect to the added oxidant. Reactions are not fast, even at $70^{\circ} \mathrm{C}$, and they proceed along 7 days. Phenyl acetaldehyde and benzaldehyde were detected and identified in all the reactions, together with minor amounts of other products such as tert-butyl benzoate or 1-phenylethane-1,2-diol. Acetonitrile was chosen as solvent, given its good performance in epoxidation reactions with TBHP [35].

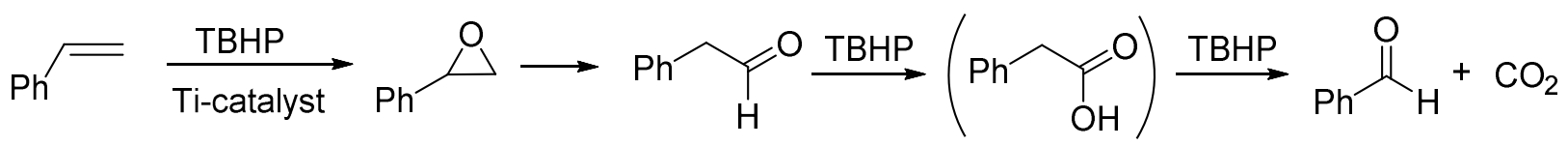

Scheme 1

Conversion represents the efficiency of the epoxidation reaction, whereas the selectivity to styrene oxide depends on the activity of the catalyst for rearrangement to phenyl acetaldehyde. As can be seen in Table 2, the homogeneous catalysts based on $\mathrm{Ti}\left(\mathrm{O}^{\mathrm{i}} \mathrm{Pr}\right)_{4}$ (entries 1-3) show a very poor selectivity. On the contrary, the grafted Ti sites on MCM-41 (entries 4-6) are less active than $\mathrm{Ti}\left(\mathrm{O}^{\mathrm{i}} \mathrm{Pr}\right)_{4}$, as shown by the lower conversion, but at the same time they are more selective, mainly due to a lower efficiency for rearrangement. The calcination temperature of MCM-41 affects the catalytic performance of the titanium sites, as Ti-MCM 750 is less efficient than the other two solids. The lower silanol density in $\mathrm{MCM}_{750}$ should produce a larger amount of monopodal sites (Figure 1), whose acidity [36] and hence the activity for epoxidation [16] must be lower than the bipodal and tripodal sites due to the lack of geometrical constrains. 
Table 2. Results of the titanium catalyzed epoxidation of styrene with TBHP. ${ }^{a}$

\begin{tabular}{|c|c|c|c|c|}
\hline Entry & Catalyst & $\begin{array}{l}\text { Conversion } 24 \mathrm{~h} \\
(\%)^{\mathrm{b}}\end{array}$ & $\begin{array}{l}\text { Styrene oxide } \\
\text { selectivity } 24 \mathrm{~h}(\%)^{\mathrm{b}}\end{array}$ & $\%$ e.e. $^{c}$ \\
\hline 1 & $\mathrm{Ti}\left(\mathrm{O}^{\mathrm{i}} \mathrm{Pr}\right)_{4}$ & $56(79)$ & $5(9)$ & - \\
\hline 2 & DET-Ti(O'Pr) $)_{4}$ & $52(75)$ & $6(9)$ & 80 \\
\hline 3 & DIPT-Ti(O'Pr $)_{4}$ & $16(40)$ & $11(9)$ & 50 \\
\hline 4 & Ti-MCM550 & $36(64)$ & $28(22)$ & - \\
\hline 5 & Ti-MCM 650 & $38(58)$ & $32(26)$ & - \\
\hline 6 & Ti-MCM ${ }_{750}$ & $23(44)$ & $29(25)$ & - \\
\hline 7 & DET-Ti-MCM550 & $68(79)$ & $44(36)$ & 55 \\
\hline 8 & DET-Ti-MCM $_{650}$ & $62(74)$ & $45(36)$ & 56 \\
\hline 9 & DET-Ti-MCM $_{750}$ & $27(54)$ & $23(32)$ & 38 \\
\hline 10 & DIPT-Ti-MCM550 & $59(64)$ & $46(26)$ & 62 \\
\hline 11 & DIPT-Ti-MCM 650 & $19(73)$ & $48(34)$ & 38 \\
\hline 12 & DIPT-Ti-MCM $_{750}$ & $26(52)$ & $42(47)$ & 45 \\
\hline
\end{tabular}

${ }^{\mathrm{a}}$ Reaction conditions: styrene (10 mmol), TBHP (6 mmol, $5.5 \mathrm{M}$ in decane), acetonitrile (10 $\mathrm{mL})$, catalyst $(0.015 \mathrm{mmol}$ of $\mathrm{Ti}), 70^{\circ} \mathrm{C}$, inert atmosphere. ${ }^{\mathrm{b}}$ Determined by GC. In parenthesis the value after 7 days. ${ }^{c}$ Determined by HPLC with a Chiralpack OD-H column.

The treatment with chiral tartrates, DET and DIPT, clearly modifies the nature of the Ti sites, as both the activity and selectivity changes. In some cases, it is clear that epoxidation is faster with the modified catalyst, in agreement with the reported behavior of epoxidation as a ligand accelerated catalytic reaction [37]. However, not all the Ti-tartrate species in solution are more active than the plain $\mathrm{Ti}\left(\mathrm{O}^{\mathrm{i}} \mathrm{Pr}\right)_{4}$, and in the case of the solid catalysts, $\mathrm{Ti}-\mathrm{MCM}_{550}$ is more prone to generate the active species, both with DET (entry 7) and DIPT (entry 10). For Ti- 
$\mathrm{MCM}_{650}$ the improvement effect is clear with DET (entry 8), but not so important with DIPT (entry 11), whereas the role of ligand is less remarkable for Ti-MCM ${ }_{750}$ with both tartrates (entries 9 and 12). The best results are then obtained with DET-Ti-MCM550, DET-Ti-MCM 650 and DIPT-Ti-MCM550, reaching conversions of styrene in the range of 59-68\% after $24 \mathrm{~h}$, with selectivities to styrene oxide of $44-46 \%$. With respect to enantioselectivity, the values obtained with the heterogeneous catalysts are moderate, in the range of $38-62 \%$ e.e.. In the only paper describing comparable catalysts and processes [18], the reported enantioselectivity was up to $32 \%$ e.e. in the epoxidation of styrene with TBHP. The different support (HMS vs MCM-41), and mainly the different solvent used in the epoxidation reaction (dichloromethane $v s$ acetonitrile) may account for the differences in the catalytic results. In fact, in our hands some tests in dichloromethane showed a much worse results, with almost no enantioselectivity at all. There is also a qualitative correlation between the catalytic activity and the enantioselectivity. Values of $55-62 \%$ e.e. are obtained in the cases of ligandaccelerated reactions, whereas values of $38-45 \%$ e.e. are obtained in reactions in which acceleration is not observed. This is in agreement with the existence of different surface species, as it happens in solution, and only when the most active one is formed in a large extension the reaction proceeds with high enantioselectivity. The enhancement in the hydrophobic character of the sites might be also invoked to explain the higher activity, as pointed in the case of silylated Ti-silica catalysts [38-40], but the qualitative correlation between activity and enantioselectivity and the similar ligand content in all the solids point to a more specific effect connected with the type of coordination of tartrate to the Ti sites instead of the general hydrophobicity effect of silylation of silica surface. It is also remarkable that the results are not far from the enantioselectivity obtained in solution, in fact DIPT-Ti$\mathrm{MCM}_{550}$ improves the result obtained with DIPT-Ti( $\left(\mathrm{O}^{\mathrm{i}} \mathrm{Pr}\right)_{4}(62$ vs 50\% e.e., entries 10 and 3), and in all cases with better selectivity to styrene oxide. 
The catalysts based on $\mathrm{MCM}_{550}$ were recovered from the reaction mixture by simple filtration, and they were reused after washing with dichloromethane. The recovery of the catalysts leads to a remarkable decrease of the styrene conversion, $10-15 \%$ after $24 \mathrm{~h}$, indicating probably the increase of diffusional resistance due to the partial blockage of the pore system. On the contrary, the enantioselectivity was maintained in moderate values after recycling, $40 \%$ e.e. with DET-Ti-MCM 550 and 56\% e.e. with DIPT-Ti-MCM 550 . This means that the supported Titartrate species is stable under the reaction conditions.

\section{Conclusions}

Ti sites in Ti-MCM, prepared by grafting of $\mathrm{Ti}\left(\mathrm{O}^{\mathrm{i}} \mathrm{Pr}\right)_{4}$ on the MCM-41 surface, can be efficiently modified with chiral tartrates, leading to surface species whose nature depends on the calcination temperature of the siliceous support. The presence of coordinated alkoxyde and esters groups, as well as non-coordinated alcohols and esters, demonstrated by solid state NMR, points to the existence of different chiral sites due to an incomplete control of the surface species. Those sites are able to activate tert-butyl hydroperoxide for the epoxidation of styrene in an enantioselective way. In fact, they are more selective than their homogeneous counterparts, which promote more efficiently consecutive reactions such as the Meinwald rearrangement of the styrene oxide and the over-oxidation of the resulting aldehyde. The enantioselectivity is moderate and qualitatively correlates with the activity of the solid, in agreement with a ligand accelerated reaction. The best result, $62 \%$ e.e., was obtained with DIPT-Ti-MCM550, showing an effect of the calcination temperature of the support and the nature of the chiral ligand. These results open the door to future improvements of this epoxidation system, with special emphasis on the control of the surface density of silanol groups to get a more homogeneous distribution of Ti species. 


\section{Acknowledgement}

Financial support from the Spanish Ministerio de Economía y Competitividad (Project CTQ2014-52367-R) and the Diputación General de Aragón (E11 Group co-financed by the European Regional Development Funds) is gratefully acknowledged. M. F. thanks the Tunisien Ministère de l'Enseignement Supérieure et la Recherche Scientifique for a shortmobility grant (bourse d'alternance).

\section{References}

[1] C. Bonini, G. Righi, Tetrahedron 58 (2002) 4981-5021.

[2] T. Katsuki in Comprehensive Asymmetric Catalysis, Vol. II; E. N. Jacobsen, A. Pfaltz, H. Yamamoto, Eds.; Springer: Heidelberg, 2000, pp. $621-648$.

[3] E. N. Jacobsen, M. H. Wu in Comprehensive Asymmetric Catalysis, Vol. II; E. N. Jacobsen, A. Pfaltz, H. Yamamoto, Eds.; Springer: Heidelberg, 2000, pp. 649 - 678.

[4] Chiral Catalysts Immobilization and Recycling; D. E. De Vos, I. F. J. Vankelecom, P. A. Jacobs, Eds.; Wiley-VCH: Weinheim, 2000.

[5] C. E. Song, S. Lee, Chem. Rev. 102 (2002) 3495-3524.

[6] J. M. Fraile, J. I. García, J. A. Mayoral, Chem. Rev. 109 (2009) 360-417.

[7] C. Baleizão, H. García, Chem. Rev. 106 (2006) 3987-4043.

[8] Q.-H. Xia, H.-Q. Ge, C.-P. Ye, Z.-M. Liu, K.-X. Su, Chem. Rev. 105 (2005) 1603-1662.

[9] L. Canali, J. K. Karjalainen, D. C. Sherrington, O. Hormi, Chem. Commun. (1997) 123124.

[10] J. K. Karjalainen, O. E. O. Hormi, D. C. Sherrington, Tetrahedron: Asymmetry 9 (1998) $1563-1575$.

[11] S. Xiang, Y. Zhang, Q. Xin, C. Li, Angew. Chem. Int. Ed. 41 (2002) 821-824. 
[12] J. M. Fraile, J. I. García, J. A. Mayoral, L. C. de Mènorval, F. Rachdi, J. Chem. Soc., Chem. Commun. (1995) 539-540.

[13] R. D. Oldroyd, J. M. Thomas, T. Maschmeyer, P. A. MacFaul, D. W. Snelgrove, K. U. Ingold, D. D. M. Wayner, Angew. Chem., Int. Ed. Engl. 35 (1996) 2787-2789.

[14] C. Cativiela, J. M. Fraile, J. I. García, J. A. Mayoral, J. Mol. Catal. A 112 (1996) 259267.

[15] M. Guidotti, C. Pirovano, N. Ravasio, B. Lázaro, J. M. Fraile, J. A. Mayoral, B. Coq, A. Galarneau, Green Chem. 11 (2009) 1421-1427.

[16] J. M. Fraile, J. I. García, J. A. Mayoral, L. Salvatella, E. Vispe, D. R. Brown, G. Fuller, J. Phys. Chem. B 107 (2003) 519-526.

[17] J. M. Fraile, J. I. García, J. A. Mayoral, E. Vispe, J. Catal. 189 (2000) 40-51.

[18] Z. Fu, D. Yin, Q. Xie, W. Zhao, A. Lv, D. Yin, Y. Xu, L. Zhang, J. Mol. Catal. A 208 (2004) 159-166.

[19] W. Zhao, Z. Hao, C. Hu, J. Li, X. Xu, Micropor. Mesopor. Mater. 112 (2008) 133-137.

[20] J. M. Fraile, J. I. García, B. Lázaro, J. A. Mayoral, Chem. Commun. (1998) 1807-1808.

[21] M. G. Finn, K. B. Sharpless, J. Am. Chem. Soc. 113 (1991) 113-126.

[22] C. Copéret, R. Chabanas, R. P. Saint-Arroman, J. M. Basset, Angew. Chem. Int. Ed. 42 (2003) 156-181.

[23] Y. Sawada, K. Matsumoto, S. Kondo, H. Watanabe, T. Ozawa, K. Suzuki, B. Saito, T. Katsuki, Angew. Chem. Int. Ed. 45 (2006) 3478-3480.

[24] K. Matsumoto, T. Oguma, T. Katsuki, Angew. Chem. Int. Ed. 48 (2009) 7432-7435. [25] X.-Y. Hao, Y.-Q. Zhang, J.-W. Wang, W. Zhou, C. Zhang, S. Liu, Micropor. Mesopor. Mater. 88 (2006) 38.

[26] C.-Y. Chen, H.-X. Li and M. E. Davis, Micropor. Mater. 2 (1993) 17. 
[27] J. S. Beck, J. C. Vartuli, W. J. Roth, M. E. Leonowicz, C. T. Kresge, K. D. Schmitt, C. T. W. Chu, D. H. Olson, E. W. Sheppard, S. B. McCullen, J. B. Higgins, J. L. Schlenker, J. Am. Chem. Soc. 114 (1992) 10834.

[28] I. Kinski, H. Gies, F. Marlow, Zeolite 19 (1997) 375.

[29] K. S. W. Sing, D. H. Everett, R. A. W. Haul, L. Moscou, R. A. Pierotti, J. Rouquérol, T. Siemieniewska, Pure Appl. Chem. 57 (1985) 603-619.

[30] R.B. Laughlin, J.D. Joannopoulos, Phys. Rev. B 16 (1977) 2942.

[31] P. G. Potvin, B. G. Fieldhouse, Tetrahedron: Asymmetry 10 (1999) 1661-1672.

[32] See supporting information of: S. F. Pedersen, J. C. Dewan, R. R. Eckman, K. B.

Sharpless, J. Am. Chem. Soc. 109 (1987) 1279-1282.

[33] M. Fadhli, I. Khedher, J. M. Fraile, J. Mol. Catal. A 410 (2015) 140-148.

[34] J. M. Fraile, N. García, J. A. Mayoral, F. G. Santomauro, M. Guidotti, ACS Catal. 5 (2015) 3552-3561.

[35] M. Guidotti, N. Ravasio, R. Psaro, G. Ferraris, G. Moretti, J. Catal. 214 (2003) 242-250. [36] J. M. Fraile, J. I. García, J. A. Mayoral, E. Pires, L. Salvatella, M. Ten, J. Phys. Chem. B 103 (1999) 1664-1670.

[37] D. J. Berrisford, C. Bolm, K. B. Sharpless, Angew. Chem. Int. Ed. Engl. 34 (1995) 10591070.

[38] T. Tatsumi, K. A. Koyano, N. Igarashi, Chem. Commun. (1998) 325.

[39] J. A. Melero, J. Iglesias, J. M. Arsuaga, J. Sainz-Pardo, P. de Frutos, S. Blazquez, J. Mater. Chem. 17 (2007) 377.

[40] M. Guidotti, I. Batonneau-Gener, E. Gianotti, L. Marchese, S. Mignard, R. Psaro, M. Sgobba, N. Ravasio, Micropor. Mesopor. Mater. 111 (2008) 39. 